\title{
A catalog of Bryophyta types deposited at the National Museum of Brazil
}

Paulo E.A.S. Câmara ${ }^{1,4}$, Micheline Carvalho-Silva ${ }^{1}$, Maria Sulamita Dias da Silva ${ }^{2}$ and Denilson Fernandes Peralta ${ }^{3}$

Received: 10 April, 2014. Accepted: 7 May, 2014.

\begin{abstract}
While visiting the Herbarium of the Botany Department of the National Museum of the Federal University of Rio de Janeiro (code, R), we located the types of 219 bryophyte names, most of which were published by Karl Müller (cited as Müller Hallensis), whose holotypes were lost during the bombing of the Berlin Herbarium in 1943. A total of 181 names (82\%) are possible candidates for lectotypification and most likely not to be found elsewhere. We also found the complete collection of Müller's "Bryologia Serrae Itatiaiae", made by Ernst Ule.
\end{abstract}

Key words: Brazil, Brotherus, Müller Hallensis, types, Ule

\section{Introduction}

Good taxonomic practice relies largely on correct location and identification of type material. However, locating and studying type specimens can be complicated for several reasons. Type specimens are often distributed over several herbaria or lost due to wars or other events. An example is the loss of the Berlin Herbarium in 1943 (Merrill 1943).

Within the realm of bryology, one of the most prolific botanists in describing new species was Karl Johan August Müller (1818-1899), from Halle, Germany (cited as Müller Hallensis). During his active years, he described many new species, probably more than any other bryologist ever. As an example, Müller (1898) described 117 new species, most from a single mountain in Brazil (Serra do Itatiaia). Unfortunately, in 1943, the Berlin Herbarium (the repository of his collections) was bombed and destroyed by fire (Merrill 1943; Hiepko 1987), a catastrophe of major proportions for the field of botany. As a consequence, many types, including some that were on loan from other herbaria and all types of Müller names housed there (many from Brazil), were lost. Since then, locating possible duplicates in other herbaria has been a major challenge and a taxonomic impediment. Many taxonomic works often have to deal with lost Müller types (e.g., Pursell 2007; Reese 1993).

The Herbarium of the Botany Department of the National Museum of the Federal University of Rio de Janeiro (code, R) is the oldest herbarium in Brazil. It was created in 1818, about the same time as when the first studies of the bryoflora of Brazil were undertaken (Martius 1818; Hooker 1818-1820; Raddi 1822). The Museum was created by King D. João VI of Portugal. For more than a century (until the 1960s), it housed the largest, and only, bryophyte collection in Brazil. It contains all relevant historical collections from Brazil, including many specimens from botanists such as Ernst Ule (1854-1915), Auguste Glaziou (1828-1906), Anders Regnell (1807-1818), Ludwig Riedel (1790-1861), and Per Dusén (1855-1926). Some of those early scientists were hired to work for the National Museum, including Ludwig Riedel, who was the first foreigner to have a permanent position at the Museum, Ernst Ule, who was the director of botany at the Museum, and Auguste Glaziou, who was the director of its public gardens. It currently houses a bryophyte collection of approximately 6015 specimens, including many types. Because the Museum underwent a long period of renovation, the collection was closed for many years. To our knowledge, no one has ever investigated the bryophyte types deposited there. Because it is now open to scientists, we were able to visit the Museum and examine the bryophyte collection. We found many types (mostly Müller Hallensis, as well as other relevant types), which we list here. Is very likely that some of the Müller Hallensis types found at the Museum are the only ones remaining after the 1943 destruction of the Berlin Herbarium and may be the best or even the only specimens available to serve as lectotypes.

\footnotetext{
${ }^{1}$ Universidade de Brasília, Departamento de Botânica. Campus Darcy Ribeiro, Brasilia, DF. Brazil. 70910-900.

${ }^{2}$ Universidade do Estado do Rio de Janeiro, UERJ. Rua São Francisco Xavier, 524, PHLC, sala 224, Maracanã, Rio de Janeiro, RJ. Brazil. $20550-013$.

${ }^{3}$ Instituto de Botânica de São Paulo. Av. Miguel Stefano, 3687, Água Funda, São Paulo, SP. Brazil. 04301-012.

${ }^{4}$ Author for correspondence: pcamara@unb.br
} 


\section{Material and methods}

We used the appropriate literature and the website of the Missouri Botanical Garden (www.tropicos.org) to locate the protologues and compare them with the label data. Because we are not providing a taxonomic revision, no taxonomic considerations are made; we did not perform any lectotypifications, synonymizations, or combinations. Taxonomic comments are provided when needed. Basionyms are presented here in alphabetical order. Protologues are not cited verbatim.

As many of the collections housed at the Museum were made by Ernst Ule, it is important to note that his collections often have two numbers, one is his personal collection number and the other refers to the exsiccata number in his Bryotheca Brasiliensis (Brotherus 1899). In the present paper, we present his collection number, using his Bryotheca Brasiliensis only when necessary. Because Müller Hallensis holotypes were destroyed in Berlin in 1943 (Hiepko 1987, 1990), all of the Müller names found that have not yet been lectotypified are here considered isotypes or isosyntypes.

\section{Results and discussion}

We found a total of 271 specimens, types of 219 names (some were duplicates), mostly of Müller Hallensis names, including the types of the genera Cladastomum Müll. Hal. and Meiotheciopsis Broth. Among those are 21 types of species endemic to Brazil. There was one holotype, as well as 130 isotypes, 99 isosyntypes (from 53 names), and 35 isolectotypes. A total of 181 names (82\%) are possible candidates for lectotypification. In addition, 105 names (47\%) have been already synonymized.

Some of the names presented here seem to have been ignored over the years, because they are mostly absent from the literature, no new combinations or synonymizations having been made. Because there have been few monographic studies of Brazilian mosses, the status of many of those names has yet to be investigated in taxonomic studies.

At the Museum, we also observed numerous original specimens of invalid or unpublished (in sched.) names, which are not presented here. We found the complete collection of Müller's "Bryologia Serrae Itatiaiae" (Müller 1898), made by Ernst Ule (1854-1915), German botanist and former Director of Botany at the Museum.

The numbers presented here may yet increase, because some dubious specimens are still under investigation. In addition, the liverworts (Marchantiophyta) have yet to be investigated.

\section{List of confirmed types found at R:}

Andreaea microphylla Müll. Hal., Bull. Herb. Boissier 6: 19. 1898. - Type: Brazil, Itatiaia, Agulhas Negras, E.Ule 1730 (isotype).
Andreaea pulvinata Müll. Hal. ex Beckett, Trans. \& Proc. New Zealand Inst. 25: 293.40. 1893. - Type: New Zealand, Rockwood, Canterbury, T.W.N. Beckett 359 (isolectotype, lectotype designated by Vitt (1980)).

Andreaea spurioalpina Müll. Hal., Bull. Herb. Boissier 6: 18. 1898. - Type: Brazil, Itatiaia, Agulhas Negras, E.Ule 1100, 1729 (isosyntypes).

Andreaea spurioalpina var. rubricalyx Müll. Hal., Bull. Herb. Boissier 6: 18. 1898. - Type: Brazil, Itatiaia, E.Ule 1727 (isotype).

Andreaea squarrosofiliformis Müll. Hal., Bull. Herb. Boissier 6: 19. 1898. -Type: Brazil, Itatiaia, E.Ule 1728 (isotype).

Aptychus lageniformis Müll. Hal., Bull. Herb. Boissier 6: 122. 1898. - Type: Brazil, Minas Gerais, Serra Ouro Preto, E.Ule 1477; Brazil, Rio de Janeiro, Serra Itatiaia, E.Ule 1885 (isosyntypes).

Barbula capillipes Broth., Hedwigia 34: 125. 1895. - Type: Brazil, Goyaz, próximo da cidade de Goyaz, E.Ule 1547 (isotype).

Bartramia declivium Müll. Hal., Bull. Herb. Boissier 6: 46. 1898. - Type: Brazil, Rio de Janeiro, Serra do Itatiaia, E. Ule 1818 (isosyntype).

Bartramia faucium Müll. Hal., Bull. Herb. Boissier 6: 47. 1898. - Type: Brazil, Rio de Janeiro, Serra do Itatiaia, E. Ule 1821 (isotype).

Bartramia pellucidirectis Müll. Hal., Bull. Herb. Boissier 6: 48. 1898. - Type: Brazil, Rio de Janeiro, Serra do Itatiaia, E. Ule 1816 (isosyntype).

Bartramia rivalis Müll. Hal., Bull. Herb. Boissier 6: 46. 1898. - Type: Brazil, Itatiaia, E.Ule 1820 (isotype).

Bartramia ulei Müll. Hal., Bull. Herb. Boissier 6: 46. 1898. - Type: Brazil, Santa Catarina, Serra Geral, E.Ule 36; Rio de Janeiro, Itatiaia, E.Ule 1819 (isosyntypes). Comments: The protologue cites two syntypes: E.Ule 662 from Santa Catarina and E.Ule 1819 from Itatiaia. Griffin III (1984) and TROPICOS lists as a syntype an E.Ule 36, from Santa Catarina; however, this number does not refer to Ernest Ule's collection number but rather the number of his "Bryophytorum Bibliotheca". There is no evidence that E. Ule 36 would correspond to E.Ule 662. We would suggest in the future the lectotypification using E.Ule 1819, as it is clearly the type material.

Brachymenium subcrinitum Paris, Index Bryol. Suppl. 40. 1900 - Type: Brazil, Rio de Janeiro, Serra Itatiaia, E.Ule 1776, 1777 (isosyntypes). Comments: On the label the name is Bryum rugosum Müll. Hal., Bull. Herb. Boissier 6: 30.1898 an illegimate name. The new name is Brachymenium subcrinitum Paris, with the same syntypes.

Brachysteleum patens Müll. Hal., Bull. Herb. Boissier 6: 108. 1898. - Type: Brazil, Rio de Janeiro, Serra do Itatiaia, E.Ule 1829 (isotype).

Brachythecium condensatissimum Müll. Hal., Hedwigia 40: 75. 1901. - Type: Brazil, Minas Gerais, Paranahyba, E.Ule 1604 (isotype). 
Brachythecium crinitirameum Müll. Hal., Hedwigia 40: 75. 1901. - Type: Brazil, Minas Gerais, Ouro Preto, E.Ule 1475 (isotype).

Brachythecium poadelphus Müll. Hal., Hedwigia 40: 76. 1901. - Type: Brazil, Minas Gerais, Caraça, E.Ule 1473 (isotype).

Brachythecium pseudosulphureum Müll. Hal., Hedwigia 40:75. 1901. - Type: Brazil, Minas Gerais, Itabira, E.Ule 1476 (isosyntype).

Braunia macrocalyx Müll. Hal., Bull. Herb. Boissier 6: 110. 1898. - Type: Brazil, Rio de Janeiro, Serra do Itatiaia, E.Ule 1848, 1849; Agulhas Negras, E.Ule 1850, 1851 (isosyntypes).

Braunia serrae Müll. Hal., Bull. Herb. Boissier 6: 111. 1898. - Type: Brazil, Santa Catarina, Serra Geral, E.Ule s.n. (isosyntype).

Braunia subincana Broth., Bih. Kongl. Svenska Vetensk.-Akad. Handl. 26 Afd. 3(7): 37. 1900. - Type: Brazil, Rio Grande do Sul, Colonia Ijuhy, Lindman 214 (isotype).

Bryum altopedunculatum Müll. Hal., Gen. Musc. Frond. 239. 1900. - Type: Brazil, Santa Catarina, Tubarão, VIII-1889, E.Ule (Bryotheca Brasiliensis 26) (isosyntype).

Bryum baenitzii Müll. Hal., Flora 71: 417. 1888. - Type: Norway, Lyngenfjord, prox. Lyngseidet, Baenitz s.n. (isotype).

Bryum crassicostatum Müll. Hal., Bull. Herb. Boissier 6: 31. 1898. - Type: Brazil, Rio de Janeiro, Serra do Itatiaia, E.Ule 1775, 1336 (isosyntypes).

Bryum duplicatum Broth., Bih. Kongl. Svenska Vetensk.-Akad. Handl. 26 Afd. 3(7): 29. 1900. - Type: Brazil, Mato Grosso, Cupim, Lindman 401 (isotype).

Bryum fabroniopsis Müll. Hal., Hedwigia 34: 127. 1895. — Type: Brazil, Goiás (city of Goiás), E.Ule 1534 (isotype).

Bryum grammocarpum Müll. Hal., Bull. Herb. Boissier 6: 32. 1898. - Type: Brazil, Rio de Janeiro, Serra do Itatiaia, E.Ule 352 (isotype).

Bryum riparioides E.B. Bartram, J. Wash. Acad. Sci. 42(6): 180. 1952. - Type: Brazil, Rio Grande do Sul, Vila Oliva, São Francisco de Paula, Sehnem 2739 (isotype).

Bryum superpensum Müll. Hal., Bull. Herb. Boissier 6: 33. 1898. - Type: Brazil, Rio de Janeiro, Serra Itatiaia, E. Ule 1770 (isotype).

Calymperes uleanum Broth., Hedwigia 34: 123. 1895. Type: Brazil, Goyaz, E.Ule 1556 (isotype).

Campylopus sehnemii E.B. Bartram., J. Wash. Acad. Sci. 42(6): 179. 1952. - Type: Brazil, Rio Grande do Sul, Montenegro, campestre, Sehnem 2310 (isotype).

Catharinea rio-grandensis Broth., Bih. Kongl. Svenska Vetensk.-Akad. Handl. 26 Afd. 3(7): 32. 1900. - Type: Brazil, Rio Grande do Sul, Silveira Martins, Lindman 210 (isosyntype).

Cladastomum ulei Müll. Hal., Bull. Herb. Boissier 6: 21. 1898. - Type: Brazil, Rio de Janeiro, Serra do Itatiaia, 1894, E.Ule 1902 (isotype). Comments: The type specimen of this species is Ule 1902, not Bryotheca Brasiliensis 201, as cited in TROPICOS.

Conomitrium generalium Müll. Hal., Hedwigia 39: 240. 1900. - Type: Brazil, Minas Gerais, Itacolumitica, E.Ule 1278 (isosyntype).

Conomitrium minutipes Müll. Hal., Hedwigia 39: 242. 1900. - Type: Brazil, Minas Gerais, Itacolumy, E.Ule 1271 (isotype).

Conomitrium perpygmaeum Müll. Hal., Hedwigia 39: 243. 1900. - Type: Brazil, Rio de Janeiro, E.Ule 1625 (isotype).

Conomitrium trichopodium Müll. Hal., Hedwigia 39: 243. 1900. - Type: Brazil, Rio de Janeiro, aqueduct de Corcovado, E.Ule 1626 (isolectotype, lectotype designated by Pursell (2007)). Rio de Janeiro, Corcovado aqueduct, E.Ule 1628 (isosyntype).

Cryphaea leptopteris Müll. Hal., Hedwigia 40: 78. 1901. - Type: Brazil, Santa Catarina, Serra Geral, Serra do Oratorio, E.Ule s.n. (Bryotheca Brasiliensis 71) (isotype). Comments: This specimen lacks a Ule collection number (only his Bryotheca Brasiliensis number), the protolgue cites E.Ule 649, but all other data on the label matches, including Müller's handwriting.

Cupressina anacamptopteris Müll. Hal., Bull. Herb. Boissier 6: 123. 1898. - Type: Brazil, Serra de Itatiaia, E.Ule 1882 (isotype).

Cupressina campaniformis Müll. Hal. Hedwigia 40: 72. 1901. - Type: Brazil, Serra dos Órgãos, E.Ule 1262, 1263; Caraça, E.Ule 1474 (isosyntypes).

Cupressina producta Müll. Hal., Bull. Herb. Boissier 6: 122. 1898. - Type: Brazil, Serra Itatiaia, E.Ule 1881 (isotype).

Daltonia uleana Müll. Hal., Bull. Herb. Boissier 6: 111. 1898. - Type: Brazil, Rio de Janeiro, Serra do Itaitiaia, E.Ule 1868 (isosyntype).

Dicranum araucarieti Müll. Hal., Hedwigia 40: 254. 1901. - Type: Brazil, Santa Catarina, Serra Geral, E.Ule s.n. (Bryotheca brasiliensis 51) (isosyntype). Comments: This specimen lacks E.Ule collection number (only his Bryotheca Brasiliensis number), the protolgue cites E.Ule 661, but all other data on label matches, including Müller's handwriting.

Dicranum brasiliense Müll. Hal., Bull. Herb. Boissier 6: 39. 1898. - Type: Brazil, Rio de Janeiro, Itatiaia, E.Ule 1906 (isolectotype, lectotype designated by Frahm (1991)). Comments: Tropicos lists the protologue as published in Bull. Herb. Boissier 6: 32.1898, when it is actually in Bull. Herb. Boissier 6: 39. 1898.

Dicranum campiadelphus Müll. Hal., Hedwigia 39: 252. 1900. - Type: Brazil, Minas Gerais, Serra do Caraça, E.Ule 1354; Minas Gerais, Ouro Preto, E.Ule 1355 (isosyntypes).

Dicranum crispatum Müll. Hal., Bull. Herb. Boissier 6: 36. 1898. - Type: Brazil, Itatiaia, E.Ule 1799 (isolectotype, lectotype designated by Frahm (1991)); E.Ule 1800 (isosyntype). 
Dicranum dicnemoides Müll. Hal., Bull. Herb. Boissier 6: 36. 1898. - Type: Brazil, Itatiaia, E.Ule 1788 (isolectotype, lectotype designated by Frahm 1991).

Dicranum dichrostis Mull. Hal., Hedwigia 39: 255. 1900. - Type: Brazil, Minas Gerais, Ouro Preto, E.Ule 1359 (isolectotype, lectotype designated by Frahm 1991).

Dicranum filicaudadum Mull. Hal., Hedwigia 39: 260. 1900. - Type: Brazil, Minas Gerais, Serra de Ouro Preto, E.Ule 1367 (isolectotype, lectotype designated by Frahm 1991).

Dicranum flaccidus Müll. Hal., Hedwigia 39: 251. 1900. Type: Brazil, Minas Gerais, Serra de Ouro Preto, E.Ule 1362 (isotype).

Dicranum gastro-alare Müll. Hal., Hedwigia 39: 256. 1900. - Type: Brazil, Minas Gerais, Serra de Caraça, E.Ule 1361 (isolectotype, lectotype designated by Frahm 1991).

Dicranum gemmatum Müll. Hal., Bull. Herb. Boissier 6: 34 . 1898. - Type: Brazil, Serra Itatiaia, E.Ule 1789 (isolectotype, lectotype designated by Frahm 1991).

Dicranum laxobasis Müll. Hal., Hedwigia 39: 252. 1900. — Type: Brazil, Minas Gerais, Caraça, E.Ule 1353 (isolectotype, lectotype designated by Frahm 1991).

Dicranum microjulaceum Müll. Hal., Bull. Herb. Boissier 6: 37. 1898. - Type: Brazil, Serra do Itatiaia, E.Ule 1796 (isotype).

Dicranum pleurocarpum Müll. Hal., Bull. Herb. Boissier 6: 35. 1898. - Type: Brazil, Itatiaia, E.Ule 1798 (isolectotype, lectotype designated by Frahm 1991).

Dicranum pseudobrachymitrium Müll. Hal., Hedwigia 39: 249. 1900. - Type: Brazil, Minas Gerais, Itatiaia, E.Ule 1803 (isolectotype, lectotype designated by Frahm 1991).

Dicranum stricticaule Müll. Hal., Bull. Herb. Boissier 6: 38 . 1898. - Type: Brazil, Rio de Janeiro, Itatiaia, E. Ule 1795 (isolectotype, lectotype designated by Frahm 1991).

Dicranum strictisetum Müll. Hal., Hedwigia, 36: 253. 1897. - Type: Brazil, Minas Gerais, Caraça, E.Ule 1357 (isolectotype, lectotype designated by Frahm 1991).

Dicranum subpenicillatum Müll. Hal., Hedwigia, 39: 257. 1897. - Type: Brazil, Minas Gerais, Caraça, E.Ule 1365 (isosyntype).

Dicranum trachynotum Müll. Hal., Bull. Herb. Boissier 6: 33. 1898. - Type: Brazil, Itatiaia, E.Ule 1801 (isotype).

Dicranum ventri-alare Müll. Hal., Hedwigia 39: 251. 1900. - Type: Brazil, Minas Gerais, Serra Ouro Preto, E.Ule 1347 (isolectotype, lectotype designated by Frahm 1991).

Diphyscium ulei Müll. Hal., Hedwigia, 36: 334. 1897. Type: Brazil, Serra dos Orgãos, E.Ule 1237 (isosyntype). Comments: The protologue does not cite any collection numbers (TROPICOS lists a syntype Ule 228). As the date and locality matches we considered it a type.

Dissodon arenarius Müll. Hal., Bull. Herb. Boissier 6: 24. 1898. - Type: Brazil, Minas Gerais, Caraça, E. Ule 1398 (isotype).

Entodon polysetus Müll. Hal., Bull. Herb. Boissier 6: 118. 1898. - Type: Brazil, Rio de Janeiro, Serra do Itatiaia, 2000 m, E.Ule 1870, 1869 (isosyntypes).
Entosthodon oligophyllus Müll. Hal., Bull. Herb. Boissier 6: 24. 1898. - Type: Brazil, Serra Itatiaia, E.Ule 1904 (isotype).

Entosthodon ouropretense Paris, Index Bryol. (ed. 2), 2: 145. 1904. - Type: Brazil, Minas Gerais, Ouro Preto, E.Ule 1310 (isotype). Comments: On the label the name is Entosthodon obtusifolius Müll. Hal., Hedwigia 39: 245. 1900, a illegitimate name, so Entosthodon ouropretense Paris is a new name but holds the same type.

Ephemerum grandifolium Müll. Hal., Bull. Herb. Boissier 6: 20. 1898. - Type: Brazil, Santa Catarina, Tubarão, Ule Bryotheca Brasiliensis 9 (probably isosyntype). Comments: The material in $\mathrm{R}$ matches the date and locality, but lacks collector number.

Ephemerum lacustre Müll. Hal., Hedwigia 34: 117. 1895. Type: Brazil, Goyaz, Tocantins river, E.Ule 1500, 1501 (isosyntypes).

Ephemerum pachyneuron Müll. Hal., Bull. Herb. Boissier 6: 20. 1898. - Type: Brazil, Itatiaia, E.Ule 1901 (isotype).

Fissidens antennidens Müll. Hal. ex Broth., Ergebn Bot. Exped. Südbras. 1901 273. 1924. - Type: Brazil, Santa Catarina, Tubarão, E.Ule 44 (isolectotype, lectotype designated by Pursell 1994).

Fissidens araucarieti Müll. Hal., Bull. Herb. Boissier 6 (2): 23. 1898. - Type: Brazil, num ribeiro da Serra do Itatiaia, E.Ule 1731 (isosyntype).

Fissidens constrictus Müll. Hal., Bull. Herb. Boissier 6: 23. 1898. - Type: Brazil, Rio de Janeiro, Serra Itatiaia, E.Ule 1733 (isolectotype, lectotype designated by Pursell 1994).

Fissidens evanescens Broth. Hedwigia 34: 120. 1895. Type: Brazil, Goyaz, E.Ule 1508 (isolectotype, lectotype designated by Pursell (1994)); E.Ule 1515, 1514, 1512, 1509 (isosyntypes).

Fissidens faucium Müll. Hal., Bull. Herb. Boissier 6: 23. 1898. - Type: Brazil, Rio de Janeiro, Serra Itatiaia, E. Ule 1734 (isolectotype, lectotype designated by Pursell (1994)), 1732, 1735 (isosyntypes).

Fissidens goyazensis Broth., Hedwigia 34: 120. 1895. - Type: Brazil, Goyaz, E.Ule 1510 (isosyntype).

Fissidens hemibryoides Müll. Hal., Hedwigia 39: 238. 1900. - Type: Brazil, Rio de Janeiro, Serra dos Órgãos 1225 (isolecotype, lectotype designated by Pursell (1994)); Minas Gerais, Ouro Preto, E.Ule 1273; Rio de Janeiro, Tijuca, E.Ule 1624, Corcovado, E.Ule 1622 (isosyntypes).

Fissidens malmei Broth., Bih. Kongl. Svenska Ventensk.-Akad. Handl. 26, Afd. III(7): 13. 1900. - Type: Brazil, Rio Grande do Sul, Porto Alegre, Lindman 44 (isotype).

Fissidens mattogrossensis Broth., Bih. Kongl. Svenska Ventensk.-Akad. Handl. 26, Afd. III(7): 14. 1900. Type: Brazil, Mato Grosso, Cuyaba, Coxipó, Lindman 351 (isotype).

Fissidens opacus Müll. Hal., Hedwigia 39: 240. 1900. - Type: Brazil, Rio de Janeiro, E.Ule 1621 (isolectotype, lectotype designated by Pursell (1994)). 
Fissidens rufinervis Müll. Hal., Hedwigia 39: 240. 1900. — Type: Brazil, Rio de Janeiro, Morro Dois Irmãos, E.Ule 1629 (isosyntype).

Fissidens schwackeanus Broth., Hedwigia 34: 119. 1895. Type: Brazil, Minas Gerais, perto de Uberaba, E.Ule 1504 (isotype).

Fissidens schwackeanus var. terrestris Broth., Hedwigia 34: 119. 1895. - Type: Brazil, Goyaz, Mossamedes. E.Ule 1513 (isotype).

Fissidens spuriolimbatus Broth., Hedwigia 34: 121. 1895. - Type: Brazil, Mossamedes, E.Ule1518 (isolectotype, lectotype designated by Pursell (2007)); E.Ule 1522 (isosyntype).

Fissidens subnutans Müll. Hal., Hedwigia 39: 239. 1900. - Type: Brazil, Rio de Janeiro, Mte. Corcovado, E.Ule 1623 (isotype).

Fissidens subradicans Broth., Hedwigia 34: 121. 1895. Type: Brazil, Goyaz, E.Ule 1506 (isolectotype, lectotype designated by Pursell (1994)); Goyaz, Mossamedes, E.Ule 1505 (isosyntype). Comments: Tropicos.org presents the mistaken opus princeps: Hedwigia 35: 121. 1896.

Garovaglia ulei Müll. Hal., Hedwigia 34: 128. 1895. - Type: Brazil, Goiás, Mossamedes, E.Ule 1620 (isotype).

Grimmia itatiaiae Müll. Hal., Bulletin de l〉Herbier Boissier 6: 109. 1898. - Type: Brazil, Rio de Janeiro, Serra do Itatiaia, E.Ule 1830 (isolectotype, lectotype designated by Muñoz (1998)).

Haplocladium flavinerve Müll. Hal., Hedwigia 40: 82. 1901. - Type: Brazil, Goyaz, Lagoa Feia, E.Ule 1597 (isotype).

Harrisonia appendiculatum Müll. Hal., Oesterr. Bot. Z. 47: 396. 1897. - Type: Brazil, Itatiaia, E.Ule 1854, 1856, $1857,1858,1859$ (isosyntypes).

Harrisonia fontinaloides Müll. Hal., Oesterr. Bot. Z. 47: 389. 1897. - Type: Brazil, Serra do Órgãos, E.Ule 1251 (isosyntype).

Harrisonia fontinaloides var. condensata Müll. Hal., Oesterr. Bot. Z. 47: 390. 1897. - Type: Brazil, Itatiaia, Agulhas Negras, E.Ule 1464 (isosyntype).

Harrisonia fontinaloides var. viridis Müll. Hal., Oesterr. Bot. Z. 47: 390. 1897. - Type: Brazil, Serra de Itatiaia, E.Ule 1889 (isosyntype).

Harrisonia pallidipila Müll. Hal., Oesterr. Bot. Z. 47: 393. 1897. - Type: Brazil, Minas Gerais, Serra do Caraça, E.Ule 1462, 1463 (isosyntypes).

Harrisonia rivularis Müll. Hal., Oesterr. Bot. Z. 47: 390. 1897. - Type: Brazil, Itatiaia, E.Ule 1871 (isotype).

Harrisonia rubiginosa Müll. Hal., Oesterr. Bot. Z. 47: 393. 1897. - Type: Brazil, Itatiaia, E.Ule 1855 (isotype).

Holomitrium seticalyx Müll. Hal., Bull. Herb. Boissier 6: 40. 1898. - Type: Brazil, Itatiaia, E.Ule 1779, 1780 (isosyntypes). Comments: The correct publication page is 40 not 41 as listed in TROPICOS.

Hookeria caudifrons Müll. Hal., Hedwigia 39: 281. 1900. — Type: Brazil, Minas Gerais, Itacolomy, E.Ule 1471, 1469 (isosyntypes). Comments: The specimen Ule 1471 has the name Hookeria leucolomoides (Nom. Nud.) written on its label.

Hookeria daltoniaecarpa Müll. Hal., Bull. Herb. Boissier 6: 112. 1898. - Type: Brazil, Rio de Janeiro, Serra do Itatiaia, E.Ule 1866 (isotype).

Hookeria glaucifolia Müll. Hal., Bull. Herb. Boissier 6: 112. 1898. - Type: Brazil, Rio de Janeiro, Serra do Itaitiaia, E.Ule 1864 (isotype).

Hookeria latifrondea Müll. Hal., Hedwigia 39: 279. 1900. — Type: Brazil, Minas Gerais, Serra de Ouro Preto, E.Ule 1472 (isotype).

Hookeria perfulva Müll. Hal., Hedwigia 39: 281. 1900. — Type: Brazil, Minas Gerais, Serra de Ouro Preto, E.Ule 1495 (isotype).

Hookeria regnelliana Müll. Hal., Hedwigia 39: 279. 1900. - Type: Brazil, Minas Gerais, região do Paranahyba, E.Ule 1590 (isotype).

Hookeria tijucae Müll. Hal., Hedwigia 39: 283. 1900. - Type: Brazil, Rio de Janeiro, Tijuca, E.Ule 1704 (isotype).

Lepidopilum caudicaule Müll. Hal., Hedwigia 39: 272. 1900. - Type: Brazil, Rio de Janeiro, Serra do Itaitiaia, E.Ule 1249 (isotype).

Leptotrichum itatiaiae Müll. Hal., Bull. Herb. Boissier 6: 41. 1898. - Type: Brazil, Rio de Janeiro, Serra do Itatiaia, E.Ule 1809, 1810 (isosyntypes).

Leptotrichum liliputanum Müll. Hal., Bull. Herb. Boissier 6: 42. 1898. - Type: Brazil, Serra Itatiaia, E.Ule 1808 (isotype).

Leucobryum clavatum var. brevifolium Broth., Ergeb. Bot. Exped. Südbras., Musci 277. 1924. - Type: Brazil, Rio de Janeiro, Tijuca, E.Ule 1630 (isosyntype).

Leucobryum goyazense Broth., Hedwigia 34: 118. 1895. Type: Brazil, Goyaz, Serra Dourada, E.Ule 1524 (isotype).

Macromitrium adnatum Müll. Hal., Bull. Herb. Boissier 6(2): 96. 1898. - Type: Brazil, Goiás, Serra Dourada, E. Ule 1558 (isotype).

Macromitrium brotheri Müll. Hal., Bull. Herb. Boissier 6(2): 97. 1898. - Type: Brazil, Goiás, E.Ule 661; Mossamedes, E.Ule 658; Goiás, Serra Dourada, E. Ule 1559/656 (isosyntypes).

Macromitrium chrysomitrium Müll. Hal., Bull. Herb. Boissier 6: 101. 1898. - Type: Brazil, Rio de Janeiro, Sera do Itatiaia, E.Ule 1836 (isotype).

Macromitrium diversifolium Broth., Hedwigia 34: 126. 1895. - Type: Brazil, Goiás, Corumbá de Goiás, E. Ule 1562 (isotype).

Macromitrium eriomitrium Müll. Hal., Bull. Herb. Boissier 6(2): 98. 1898. - Type: Brazil, Serra Itatiaia, E. Ule 1834 (isotype).

Macromitrium lampromitrium Müll. Hal., Bull. Herb. Boissier 6: 101. 1898. - Type: Brazil, Rio de Janeiro, E.Ule 1837 (isotype).

Macromitrium podocarpi Müll. Hal. var. falcifolium Müll. Hal., Bull. Herb. Boissier 6(2): 96. 1898. - Type: Brazil, Minas Gerais, Caraça, E. Ule 1408 (isotype). 
Macromitrium subpycnangium Müll. Hal., Bull. Herb. Boissier 6: 100. 1898. - Type: Brazil, Rio de Janeiro, Tijuca, XII-1893, E.Ule 1671 (isotype).

Macromitrium substrictifolium Müll. Hal., Bull. Herb. Boissier 6: 98. 1898. - Type: Brazil, Rio de Janeiro, Tijuca, E.Ule 1672 (isotype).

Macromitrium undatum Müll. Hal., Bull. Herb. Boissier 6: 97. 1898. - Type: Brazil, Rio de Janeiro, Serra do Itatiaia, E.Ule 1832 (isotype).

Meteorium serricolum Müll. Hal., Bull. Herb. Boissier 6: 116. 1898. - Type: Brazil, Minas Gerais, Serra do Ouro Preto, E.Ule 1453; Minas Gerais, Serra do Itacolomy, E.Ule 1454; Rio de Janeiro, Serra do Itatiaia, E.Ule 1845 (isosyntypes).

Mielichhoferia grammocarpa Müll. Hal., Bull. Herb. Boissier 6:27. 1898. - Type: Brazil, Itatiaia, E. Ule 1774 (isotype).

Mnium acuminatissimum Müll. Hal., Hedwigia 39: 248. 1900. - Type: Brazil, Rio de Janeiro, Tijuca, E.Ule 1644 (isotype).

Mnium rhynchomitrium Müll. Hal., Hedwigia 39: 247. 1900. - Type: Brazil, Rio de Janeiro, Corcovado, E.Ule 1639, 1640; Minas Gerais, Caraça, E.Ule 1312; Minas Gerais, Ouro Preto, E.Ule 1311 (isosyntypes).

Moenkemeyera abrupta Broth., Bih. Kongl. Svenska Vetensk.-Akad. Handl. 26 Afd. 3(7): 14. 1900. - Type: Paraguay. Santo Antonio, colonia "Elisa" ad arbores, Lindman 221 (isotype).

Moenkemeyera alomifolia Müll. Hal., Hedwigia 39: 236. 1900. - Type: Brazil, Minas Gerais, Serra do Itacolumy, E. Ule s.n. (isotype).

Neckera araucarieti Müll. Hal., Bull. Herb. Boissier 6: 115. 1898. - Type: Brazil, Rio de Janeiro, Serra do Itatiaia, E.Ule 1847 (isosyntype).

Octoblepharum perforatum Müll. Hal., Hedwigia 34: 119. 1895. - Type: Brazil, Goyaz, Corumba, E.Ule 1546 (isotype).

Octoblepharum rhaphidostegium Müll. Hal. in Brotherus, Hedwigia 34: 119. 1895. —Type: Brazil, Goiás, Serra dos Pirineus, E. Ule 1525 (isotype).

Octoblepharum rupestre Müll. Hal. ex Broth., Ergebn. Bot. Exped. Südbras., 1901. 279. 1924. - Type: Brazil, Minas Gerais, Ouro Preto, E.Ule 1392 (isotype).

Orthodontium arenarium Müll. Hal., Bull. Herb. Boissier 6: 29. 1898. - Type: Brazil, Minas Gerais, Caraça, E.Ule 1399 (isotype).

Orthostichella subtenuis Müll. Hal., Hedwigia 40: 88. 1901. - Type: Brazil, Rio de Janeiro, Corcovado, E.Ule 1255 (isosyntype).

Orthotrichum araucarieti Müll. Hal. ex Broth., Denkschr., Akad. Wiss. Wien Math. Nat. Kl. 83: 287. 1924. - Type: Brazil, Santa Catarina, Serra Geral, 1890, E.Ule Bryotheca Brasiliensis 20 (isolectotype, lectotype designated by Lewinsky (1987)).

Papillaria bipinnata Müll. Hal., Bull. Herb. Boissier 6: 117. 1898. - Type: Brazil, Serra de Itatiaia, E. Ule 1843 (isotype).
Papillaria capillicuspis Müll. Hal., Hedwigia 40: 96. 1901. - Type: Brazil, Rio de Janeiro, Corcovado, E.Ule 1686 (isotype).

Papillaria rutenbergiacea Müll. Hal., Hedwigia 40: 90. 1901. — Type: Brazil, Rio de Janeiro, Tijuca, E.Ule 1697 (isotype).

Papillaria viridata Müll. Hal. ex Broth., Bih. K. Svenska Vetensk. Akad. Handl. 26, 3(7): 42. 1900. — Type: Paraguay, ad truncos arborum silvae montis Santo Thomas, C.A.M. Lindman 256 (isotype).

Philophyllum bromeliae Müll. Hal., Bull. Herb. Boissier 6: 123. 1898. - Type: Brazil, Rio de Janeiro, Serra do Itaitiaia, E.Ule 1860 (isolectotype, lectotype designated by Buck (1992)).

Physcomitrium capillipes Müll. Hal. ex Broth. Hedwigia 34: 127. 1895. - Type: Brazil, Goyaz, rio Paranahyba, E.Ule 1532 (isotype).

Physcomitrium falcifolium Müll. Hal. ex Broth. Hedwigia 34: 127. 1895. - Type: Brazil, Goyaz, Lagoa Feia, E.Ule 1531 (isotype).

Physcomitrium lindmannii Broth., Bih. Kongl. Svenska Vetensk.-Akad. Handl. 26 Afd. 3(7): 26. 1900. - Type: Brazil, Rio Grande do Sul, Porto Alegre, Lindman 45 (isotype).

Plagiothecium lepidopiladelphus Müll. Hal., Bull. Herb. Boissier 6: 119. 1898. - Type: Brazil, Rio de Janeiro, Serra do Itatiaia, E.Ule 1862 (isolectotypes, lectotype designated by Buck \& Ireland (1989)); Rio de Janeiro, Serra do Itatiaia, E.Ule 1861 (isosyntype).

Plagiothecium meteoriaceum Müll. Hal., Bull. Herb. Boissier 6: 120. 1898. - Type: Brazil, Rio de Janeiro, Serra do Itatiaia, E.Ule 1884 (isotype).

Polytrichum itatiaiae Müll. Hal., Bull. Herb. Boissier 6: 25. 1898. - Type: Brazil, Rio de Janeiro, Serra do Itatiaia, E.Ule 1764 (isotype).

Porotrichum minutistolo Müll. Hal., Bull. Herb. Boissier 6: 114. 1898. - Type: Brazil, Rio de Janeiro, Serra do Itatiaia, E.Ule 1886 (isotype). Comments: This specimen (Ule 1886) is also cited as type of Porotrichum capillistolo Müll.Hal. Bull. Herb. Boissier 6:113.1898. No plant bearing this name was found in $\mathrm{R}$.

Porotrichum olidum Müll. Hall., Bull. Herb. Boissier 6: 114. 1898. - Type: Brazil, Rio de Janeiro, Serra do Itatiaia, E.Ule 1914 (isotype).

Porotrichum suspectum Müll. Hal., Hedwigia 39: 287. 1900. - Type: Brazil, Rio de Janeiro, Corocovado, E.Ule 1698 (isotype).

Psilopilum ulei Broth ex Müll. Hal., Bull. Herb. Boissier 6: 119. 1898. - Type: Brazil, Rio de Janeiro, Serra do Itatiaia, E.Ule 1905 (isotype).

Pterobryon subangustifolium Müll. Hal., Bull. Herb. Boissier 6: 114. 1898. - Type: Brazil, Rio de Janeiro, Tijuca, E.Ule 1703 (isotype).

Ptychomnion fruticetorum Müll. Hal., Bull. Herb. Boissier 6: 126. 1898. - Type: Brazil, Rio de Janeiro, Serra Itatiaia, E.Ule 1915 (isosyntype). 
Pungentella spuriopatens Müll. Hal., Hedwigia 40: 74. 1901. - Type: Brazil, Rio de Janeiro, Pico do Papagaio. E.Ule 1709 (isotype).

Raphidostegium panduraefolium Broth., Bih. Kongl. Svenska Vetensk.-Akad. Handl. 26 afd. 3(7): 44. 1900. - Type: Brazil, Rio Grande do Sul, Lindman 200 (isotype).

Rhynchostegium microthamnioides Müll. Hal., Bull. Herb. Boissier 6: 124. 1898. - Type: Brazil, Rio de Janeiro, Serra do Itatiaia, E.Ule 1879 (isotype).

Schlotheimia gracilescens Broth., Bih. K. Svensk. Vetensk.-Akad. Handl. 26, 3(7): 23--24. 1900. - Type: Brazil, Rio Grande do Sul, São Leopoldo, Hamburger, C.A. Lindman 107 (isotype).

Schlotheimia grammocarpa Müll. Hal., Bull. Herb. Boissier 6(2): 102--103. 1898. - Type: Brazil, Rio de Janeiro, Serra Itatiaia, E. Ule 1839 (isotype).

Schlotheimia horridula Müll. Hal., Bull. Herb. Boissier 6(2): 105-106. 1898. - Type: Brazil, Minas Gerais, Caraça, E. Ule 1422 (isotype).

Schlotheimia macrospora Müll. Hal., Bull. Herb. Boissier 6: 103. 1898. - Type: Brazil, Minas Gerais, Itabira do Campo, E.Ule 1424, 1423, 1416; Caraça, E.Ule 1421; Ouro Preto, E.Ule 1419 (isosyntypes).

Schlotheimia pseudoaffinis Müll. Hal., Bull. Herb. Boissier 6(2): 104. 1898. - Type: Brazil, Serra Itatiaia, E. Ule 1833 (isotype).

Schlotheimia robusticuspis Müll. Hal., Bull. Herb. Boissier 6(2): 106--107. 1898. - Type: Brazil, Minas Gerais, Serra Itatiaia, E. Ule 1417, 1840 (isosyntypes).

Seligeria itatiaiae Müll. Hal., Bull. Herb. Boissier 6: 45. 1898. - Type: Brazil, Rio de Janeiro, Serra do Itatiaia, E.Ule 1807 (isotype).

Sematophyllum cataractae W.R. Buck, Brittonia 35: 328. 1983. - Type: Brazil, Santa Catarina, Serra Geral, E.Ule 92 (isotype).

Sigmatella leptosquarrosa Müll. Hal., Hedwigia 34: 129. 1895. - Type: Brazil, Goyaz, Mossamedes, E.Ule 1613, 1614 (isosyntypes).

Sigmatella olida Müll. Hal., Hedwigia 40: 69. 1901. - Type: Brazil, Rio de Janeiro, Dois Irmãos, E.Ule 1723 (isotype).

Sphagnum carneum Müll. Hal. \& Warnst., Hedwigia 36: 145. 1897. - Type: Brazil, Minas Gerais, Ouro Preto, E. Ule 1289 (isosyntype).

Sphagnum densum Müll. Hal. \& Warnst., Hedwigia 36: 147. 1897. - Type: Brazil, nos rochedos da Serra de Itatiaia, E.Ule 1743 (isotype).

Sphagnum ellipticum Müll. Hal. \& Warnst., Hedwigia 36: 165. 1897. - Type: Brazil, em rochedos da Serra do Itatiaia, E.Ule 1752 (isotype).

Sphagnum itacolumites Müll. Hal., Hedwigia 36: 172. 1897. - Type: Brazil, Minas Gerais, Itacolomi, E.Ule 1302 (isotype).

Sphagnum itatiaiae Müll. Hal., Hedwigia 36: 146. 1897. — Type: Brazil, Serra Itaitiaia, E.Ule 1742; Agulhas Negras, Serra Itaitiaia, E.Ule 1741 (isosyntypes).
Sphagnum laceratum Müll. Hal., Hedwigia 36: 149. 1897. - Type: Brazil, Minas Gerais, Serra do Caraca, E.Ule 1294 (isotype).

Sphagnum lindmanii Warnst., Bot. Centralbl. 76: 418. 1899. - Type: Paraguay, Villa Rica, Lindman 263 (isosyntype). Sphagnum longistolo Müll. Hal., Hedwigia 36: 169. 1897. - Type: Brazil, em lugares humidos, Serra dos Órgãos, E.Ule 1227 (isotype).

Sphagnum minutulum Müll. Hal., Hedwigia 36: 166. 1897. - Type: Brazil, em pantanos da Serra Itatiaia, E.Ule 1749 (isotype).

Sphagnum mirabile Müll. Hal., Hedwigia 36: 161. 1897. Type: Brazil, Minas Gerais, Caraca, E.Ule 1287 (isotype).

Sphagnum ouropretense Müll. Hal. \& Warnst., Hedwigia 36: 172. 1897. - Type: Brazil, Minas Gerais, Ouro Preto, E.Ule 1288 (isotype).

Sphagnum oxyphyllum var. nanum Müll. Hall. \& Warnst, Hedwigia 36: 150. 1897. - Type: Brazil, Rio de Janeiro, Serra do Itatiaia, E.Ule 1744, 1903 (isosyntypes).

Sphagnum paranae Warnst., Allg. Bot. Z. Syst. 11: 97.1905. - Type: Brazil, Parana, Porto D. Pedro II, Dusén 4402 (isotype).

Sphagnum pseudoacutifolium Müll. Hal. \& Warnst., Hedwigia 36: 148. 1897. - Type: Brazil, Rio de Janeiro, Serra do Itatiaia, E.Ule 1745 (isotype).

Sphagnum pumilum Müll. Hal. \& Warnst., Hedwigia 36: 163. 1897. - Type: Brazil, em rochedos das Agulhas Negras na Serra de Itatiaia, E.Ule 1750 (isotype).

Sphagnum ramulinum Warnst., Bot. Centralbl. 78: 4. 1898. - Type: Brazil, Minas Gerais, Serra do Ouro Preto, E.Ule 1304 (isotype).

Sphagnum rotundatum Müll. Hal. \& Warnst., Hedwigia 36: 162. 1897. - Type: Brazil, Rio de Janeiro, Serra do Itatiaia, E. Ule 1760 (isotype).

Sphagnum rotundifolium Müll. Hal. \& Warnst., Hedwigia 36: 159. 1897. - Type: Brazil, Rio de Janeiro, Serra do Itatiaia, E.Ule 1756 (isotype).

Sphagnum subovalifolium Müll. Hal. \& Warnst., Hedwigia 36: 162. 1897. - Type: Brazil, Rio de Janeiro, Serra do Itatiaia, E.Ule 1754 (isotype).

Sphagnum subundulatum Müll. Hal. \& Warnst., Hedwigia 36: 152. 1897. - Type: Brazil, Minas Gerais, Serra do Ouro Preto, E.Ule 1298 (isotype).

Sphagnum trigonum Müll. Hal. \& Warnst., Hedwigia 36: 158. 1897. - Type: Brazil, Rio de Janeiro, Tijuca, E.Ule 1634, 1635 (isosyntype).

Sphagnum trigonum var. laxifolium Warnst., Hedwigia 36: 159. 1897. - Type: Brazil, Rio de Janeiro, E.Ule 1632 (isotype).

Sphagnum vesiculare Müll. Hal. \& Warnst., Hedwigia 36: 173. 1897. - Type: Brazil, Minas Gerais, Itacolumy, E.Ule 1301 (isotype).

Stereophyllum lindmanii Broth., Bih. K. Svenska Vet. Akad. Handl. 26 Afd. 3(7): 50. 1900. - Type: Paraguay, Santo Thomas, Lindman 244 (isolectotype, lectotype designa- 
ted by Ireland \& Buck (1994)); Santo Antonio, Lindman 239 (isosyntype). Comments: There is a mistake on Tropicos and also on Ireland \& Buck (1994), the correct page is 50 (not 150).

Syrrhopodon arenarius Müll. Hal., Hedwigia 39: 262. 1900.

- Type: Brazil, Minas Gerais, Caraça, Ule 1387 (isolectotype, lectotype designated by Reese (1993)); Serra Ouro Preto, E.Ule 1385 (isosyntype).

Syrrhopodon brotheri Müll. Hal., Hedwigia 39: 266.1900. - Type: Brazil, Goias, Pr. Massamedes, E.Ule 1542 (isotype).

Syrrhopodon calochlorus Müll. Hal., Hedwigia 39: 263. 1900. - Type: Brazil, Rio de Janeiro, Tijuca, E.Ule 1669 (isolectotype, lectotype designated by Reese (1993)).

Syrrhopodon cymbifolius Müll. Hal., Hedwigia 39: 262:1900. - Type: Brazil, Goyaz, flum. Corumba, E.Ule 1571 (isolectotype, lectotype designated by Reese (1993)).

Syrrhopodon goyazensis Broth., Hedwigia 34: 122. 1895. Type: Brazil, Goyaz, Passo Tempo, E.Ule 1540 (isotype). Syrrhopodon laeviusculus Broth., Hedwigia 34: 122. 1895. - Type: Brazil, Goiás, Corumbá, E.Ule 1544 (isolectotype, lectotype designated by Reese (1993); E.Ule 1545 (isosyntype).

Syrrhopodon persordidus Müll. Hal., Hedwigia 39: 264. 1900. - Type: Brazil, Minas Gerais, Serra de Ouro Preto, E.Ule 1383 (isolectotype, lectotype designated by Reese (1993)).

Syrrhopodon rhizogonioides Müll. Hal., Hedwigia 39: 266. 1900. - Type: Brazil, Minas Gerais, Caraça, E.Ule 1389 (isolectotype, lectotype designated by Reese (1993)).

Syrrhopodon rosulatus Müll. Hal., Hedwigia 39: 264. 1900. - Type: Brazil, Rio de Janeiro: Tijuca, E.Ule 1666 (isolectotype, lectotype designated by Reese (1993)).

Syrrhopodon rubicundus Müll. Hal., Hedwigia 39: 263. 1900. - Type: Brazil, Minas Gerais: Serra de Caraca, E.Ule 1379 (isolectotype, lectotype designated by Reese (1993).

Syrrhopodon terebellatulus Müll. Hal., Hedwigia 39: 264. 1900. - Type: Brazil, Rio de Janeiro: Tijuca, E.Ule 1664 (isotype). Comments: This specimen is an isotype cited by Müller (1900), however Reese (1993) lectotypified the species with another specimen: E.Ule s.n.

Systegium mollifolium Müll. Hal., Hedwigia 34: 117. 1895. - Type: Brazil, Goyaz, close to Alicuns, E.Ule 1499 (isotype).

Taxicaulis afflictus Müll. Hal., Hedwigia 40: 68. 1901. Type: Brazil, Goyaz, close to Mossamedes, E.Ule 1616 (isotype).

Taxicaulis fabroniiformis Müll. Hal., Hedwigia 40: 69. 1901. - Type: Brazil, Goyaz, close to Mossamedes, E.Ule 1617 (isotype).

Taxicaulis micro-plumosus Müll. Hal., Hedwigia 40: 68. 1901. - Type: Brazil, Minas Gerais, Ouro Preto, E.Ule 1486 (isotype).

Taxicaulis percondensatus Müll. Hal., Hedwigia 40: 66. 1901. - Type: Brazil, Goyaz, close to Mossamedes, E.Ule 1618 (isotype).
Taxicaulis sapricola var. minor Müll. Hal., Hedwigia 40: 68. 1901. - Type: Brazil, Rio de Janeiro, Corcovado, E.Ule 1722 (isotype).

Taxiphyllum machrisianum H.A. Crum, Los Angeles Co. Mus. Contr. Sci. 18: 5. f. 1-4. 1957. - Type: Brazil, Goiás, Chapada dos Veadeiros, Dawson 14743a (holotype).

Tayloria ulei Müll. Hal., Hedwigia 34: 126. 1895. - Type: Brazil, Goiás, na região do Rio Paranahyba, E.Ule 1555 (isotype).

Tetraplodon itatiaiae Müll. Hal., Bull. Herb. Boissier 6: 25. 1898. - Type: Brazil, Rio de Janeiro, Serra do Itatiaia, E.Ule 1763 (isotype).

Thamnium flagellatum Müll. Hal., Bull. Herb. Boissier 6: 119. 1898. - Type: Brazil, Rio de Janeiro, Serra do Itatiaia, E.Ule 1883 (isotype).

Trematodon aureus Müll. Hal. ex G. Roth, Aussereur. Laubm. 278. 1911. - Type: Brazil, Santa Catarina, Tubarão, E.Ule (Bryotheca Brasiliensis 15) (isotype).

Trematodon brevifolius Broth. ex Müll. Hal., Bull. Herb. Boissier 6: 44. 1898. - Type: Brazil, Rio de Janeiro, E.Ule 1815 (isotype).

Trematodon heterophyllus Müll. Hal., Hedwigia 34: 11. 1895. - Type: Brazil, Minas Gerais, Serra do Caraca, E.Ule 1425 (isotype).

Trematodon pauperifolius Müll. Hal., Bull. Herb. Boissier 6: 45. 1898. - Type: Brazil, Minas Gerais, Serra do Itacolomy, E.Ule 1426 (isotype). Comments: The protologue says, "Itabira do Campo" as locality and the label "Itacolomy". However the collection number and date match.

Trismegistia breviflagellosa Mül. Hal., Hedwigia 40: 74. 1901. - Type: Brazil, Minas Gerais, Serra Ouro Preto, E.Ule 1489 (isotype).

Trismegistia fluminicola Mül. Hal., Hedwigia 40: 74. 1901. Type: Brazil, Goiás, Rio das Almas, E.Ule 1607 (isotype).

Trismegistia itatiaiae Müll. Hal., Bull. Herb. Boissier 6: 121. 1898. - Type: Brazil, Serra Itatiaia, E.Ule 1917 (isosyntype).

Trismegistia tereticaulis Müll. Hal., Bull. Herb. Boissier. 6: 121. 1898. - Type: Brazil, Serra de Itatiaia, E.Ule 1887 (isotype).

Vesicularia oedicarpa Müll. Hal., Hedwigia 40: 62. 1901. Type: Brazil, Goyaz, E.Ule 1602 (isotype).

Vesicularia paranahybae Müll. Hal., Hedwigia 40: 63. 1901. - Type: Brazil, Minas Gerais, Paranahyba. E.Ule 1603 (isotype).

Zygodon araucariae Müll. Hal., Bull. Herb. Boissier 6: 95. 1898. - Type: Brazil, Rio de Janeiro, Serra do Itatiaia, E.Ule 1918 (isosyntype).

Zygodon capillicaulis Müll. Hal., Bull. Herb. Boissier 6: 94. 1898. - Type: Brazil, Minas Gerais, Serra Itacolomy, E.Ule 1394 (isosyntype). Comments: The protologue says Itabira Hills and the label Itacolomy Hills. However everything else matches including collector number. Also the isosyntype E. Ule 1395 was also found but with an empty packet. 
Zygodon dives Müll. Hal., Bull. Herb. Boissier 6: 94. 1898. Type: Brazil, Minas Gerais, Serra de Ouro Preto, E.Ule 1396; Rio de Janeiro, Serra do Itatiaia, E. Ule 1911, 1822 (isosyntypes). Comments: These specimens are isosyntypes cited by Müller (1898), however Calabrese (2006) has lectotypified another specimen: Dusén s.n.

\section{Acknowledgments}

This study received financial support from the Herbário Virtual das Plantas e Fungos (Virtual Herbarium of Plants and Fungi), which is one of the Brazilian Institutos Nacionais de Ciência e Tecnologia (INCTs, National Institutes of Science and Technology). We thank all the faculty and staff of the Herbarium of the Botany Department of the National Museum of the Federal University of Rio de Janeiro, in particular Dr. Luci de Senna Valle, Dr. Mariângela Menezes, Dr. Andrea Costa and Vera Martins, for providing access to the collection. We are especially grateful to Dr. Leonor Costa Maia, of the INCT. We dedicate this paper to those in the past, who are no longer but live again.

\section{References}

Brotherus, V. 1899. Ule, E. Bryotheca brasiliensis, herausgegeben von Dr. C.H. Brotherus in Helsingfors, bestimmt von Carl Müller, C.H. Brotherus und C. Warnstorf. Hedwigia 38(1): 57-59.

Buck, W. 1992. A revision of Philophyllum (Hookeriales). The Bryologist 95: 334-337.

Buck, W. R. \& Ireland, R. R. 1989. Plagiotheciaceae. Flora Neotropica Monograph 50: 1-22.

Calabrese, G.M. 2006. A taxonomic revision of Zygodon (Orthotrichaceae) in southern South America. The Bryologist 109: 453-509.
Frahm, J.P. 1991. Dicranaceae: Campylopodioideae, Paraleucobryoideae. Flora Neotropica Monograph 54: 1-238.

Griffin III, D. 1984. Breutelia in Brazil with notes on the occurrence of the genus in the New World. Journal of the Hattori Botanical Laboratory 57: 83-95.

Hiepko, P. 1987. The collections of the Botanical Museum Berlin-Dahlem (B) and their history. Englera 7: 219-252.

Hiepko, P. 1990. Die Sammlungen des Botanischen Museums Berlin-Dahlem und ihre Geschichte. In: Schnarrenberger, C, Scholz, H. (ed.) Geschichte der Botanik in Berlin. Colloquium Verlag, Berlin.

Hooker, W.J. 1818-1820. Musci Exotici. 2 vols. London.

Ireland, R.R. \& Buck, W.R. 1994. Stereophyllaceae. Flora Neotropica Monograph 65: 1-49.

Lewinsky, J. 1987. Orthotrichum (Orthotrichaceae) in South America 2. Taxonomic revision of the taxa with superficial stomata. Memoirs of The New York Botanical Garden 45: 326-370.

Martius, C.F.P. 1818. Icones plantarum cryptogamicarum, quas in itinere annis 1817-1820 per Brasiliam jussu et auspiciis Maximiliani Josephi I. Bavariae regis augustissimi instituto collegit et descripsit. Monachi. 138 p.

Merrill, E.D. 1943. Destruction of the Berlin Herbarium. Science 98(2553): 490-491.

Müller, C. 1898. Bryologia Serrae Itatiaiae (Minas Gerais Brasiliae) adjectus nonnulis speciobus affinibus regionum vicinarum. Bulletin de la Herbier Bossier 6: 18-48.

Müller, C. 1900. Genera Muscorum Frondosorum vi +474 pp. Eduard Kummer, Lepizig.

Muñoz, J. 1998. Materials toward a revision of Grimmia (Musci, Grimmiaceae): nomenclature and taxonomy of Grimmia longirostris. Annals of the Missouri Botanical Garden 85: 352-363.

Pursell, R.A. 1994. Taxonomic notes on Neotropical Fissidens. The Bryologist 97: 253-271.

Pursell, R.A. 2007. Fissidentaceae. Flora Neotropica Monograph 101: $1-279$.

Raddi, G. 1822. Crittogame brasiliane racclote e descritte. Memoire dela Societá Italiana delle Scienze residente in Modena 19: 27-57.

Reese, W.D. 1993. Calymperaceae. Flora Neotropica Monograph 58: $1-102$.

Vitt, D.V. 1980. A comparative study of Andreaea acutifolia, A. mutablis, and A. rupestris. New Zealand Journal of Botany 18: 367-377. 